Aus dem Anatomischen Institut der Universität Berlin.

\title{
Beiträge zum Studium des Zentralnervensystems der Wirbeltiere.
}

\section{Ein Faserzug am Boden des Recessus praeopticus (Tractus praeopticus) bei den Amphibien.}

Von Dr. med. Paul Röthig.

Hierzu Tafel II.

Das Yaterial der vorliegenden Arbeit umfasst von den Anuren Rana und Bufo, von den Urodelen Spelerpes fuscus, Cryptobranchus japonicus, Necturus maculatus und Sirena licertina; sie hat zum Gegenstand einen Faserzug, der am Boden des Recessus praeopticus in sagittaler Richtung kaudalwarts zieht und sich unmittelbar oberhalb der postchiasmatischen Kreuzungen verliert. Dieser Faserzug, der seiner Lage entsprechend Tractus praeopticus genannt wird, ist besonders stark entwickelt bei der Kröte. Die Abbildungen $1-5$ auf Taf. II zeigen ihn auf einer Reihe kaudalwärts aufeinander folgender Frontalschnitte des Gehirns von Bufo und in Fig. 6 auf einem Längsschnitt vom gleichen Material.

Die Fig. 1 (Taf. II) stellt einen Durchschnitt durch den frontalen Teil des Recessus praeopticus dar; umgeben wird dieser von den Zellen des von C. L. Herrick so genannten Nucleus praeopticus. Am Boden dieses Recessus erblickt man den Anfangsteil der mit der Weigertschen Markscheidenfärbung blau gefärbten Fasern des Tractus praeopticus. Schreiten wir in der Serie kaudalwarts vor, so weitet sich ventral der Recessus aus (Fig. 2, Taf. II), sein Boden wölbt sich spornartig in das Lumen des Recessus vor und enthălt im Innern dieser Hervorwölbung die eng aneinander gelagerten Tractus praeopticus-Fasern. Nach oben setzt sich durch einen Spalt der Recessus in Verbindung mit dem Hohlraum des Zwischenhirns. Er wird wieder umgeben von den Zellen des Nucleus praeopticus, an die ganz dorsal die Fasern des medialen Vorderhirnbündels grenzen. Auf Fig. 3 der 
gleichen Tafel befinden wir uns im Beginn des Chiasma opticum. An dasselbe grenzt von dorsal her der Boden des kaudalen Ausliuters unseres Recessus, und zwar ist auch hier dieser Boden halbkugelförmig nach oben in denselben vorgewölbt. In ihm liegt wieder der Durchschnitt des Tractus praeopticus, der in dieser Gegend dentlich in żwei Bündel zerfüllt. Weiter nach linten legen sich diese eng aneinander (Fig. 4, 'Taf. II): sie befinden sich in der Mitte des Bodens des Hohlranmes des Diencephalon oberhalb des Chiasma. Der Ventrikel ist umgeben voll den Auslaufern des Zellarcales des Nucleus praeopticus, an denen man hier die beiden Abteilungen der vorigen Figur, nämlich die C'ellulae magnie und die Grundzellen des Kernes nicht mebr unterscheiden kann, hier besteht vielmehr der Nucleus praeopticus aus gleichartigen Zellen. Seitlich grenzen an den Kern die Durchschnitte des medialen und lateralen Vorderhimbündels. Während bis hierher der Tractus praeopticus als isolierter Zug deutlich zu unterscheiden war, ist dies weiter kaudal nicht melır möglich; dort verliert er sich allmählich in dem Fasergewirr am Boden des Ventriculus diencephali und oberhalb der postchiasmatischen Kreuzungen, wie die Fig. ’́ (Taf. II) zeigt. Dort sieht man dorsal den Hohlraum des Zwischenhirns, ventral den des Hypothalimus und zwischen beiden die Querzüge der postchiasmatischen Kreuzungen und oberhalb letzterer Faserdurchschnitte, zwischen denen sich unser Tractus verliert. Dieser ganze eben geschilderte Verlauf zeigt sich auch auf dem Sagittalschnitt in Fig. 6 ('Taf. II), all der man ebenfalls streckenweise eine Scheidung des Faserzuges in zwei Bündel bemerken kann.

Was das Vorkommen dieses Tractus praeopticus bei anderen Amphibien betrifft, so kann ich auf Grund meines Materiales folgendes aussagen: Er ist ausser bei der Kröte auch bei Rana nachweisbar; hier aber nur auf ganz kurze Strecken bin und von sehr geringer Entwicklung. Bei Spelerpes fuscus habe ich ihn nicht beobachten können, dagegen, allerdings auch hier sehr schwach ausgebildet, bei Sirena lacertina und bei Necturus maculatus. Eine mächtigere Ausbildung, die der bei Bufo nahe kommt, zeigt er bei Cryptobranchus japonicus. Hier zerfallt er nach den Befunden auf einer Frontalserie in eine Reihe sagittal verlaufender feiner Fasern. Ab und zu sieht man zwischen ihnen am Boden des Recessus praeopticus quer verlaufende Fasern. 
In der Literatur habe ich bisher bei dell Amphibien den voll mir beschriebenen $\mathrm{Zug}$ nicht erwahnt gefunden. Was ein Blick auf die anderen Wirbeltierklassen betrifft, so erscheint es mir denkbar, dass er dem von Ka pers so genannten Tractus praethal. cinereus entspricht. Ferner habe ich selbst in meiner Untersuchung über die Riechbahnen, Septum und Thalamus bei Didelphis marsupialis (Abh. Senckenberg. Naturforsch. Ges., Bd. 31, Heft 1, 1909) ein Fasersystem beschrieben, das der Lage nach mit dem Tractus praeopticus vergliclien werden kann. Es wurde dort Fasciculus supraopticus genannt und den Fasern des zentralen Graues des Sehhïgels zugerechnet. Dieser Fasciculus supraopticus verläuft dort im Boden des Recessus opticus dicht oberhalb des Opticus, nimmt seinen Anfang jederseits ans einer Zellanhäufung, dem Ganglion supraopticum frontale, und verliert sich weiter kaudal in Zellanhitufungen, die rechts und links oberhalb des Chiasma neben dem dritten Ventrikel liegen und Ganglia supraoptica caudalia heissen. Das hintere Ende des Fasciculus supraopticus wird untermischt und zum 'Teil verdeckt durch die Kuppe der Decussatio supraoptica dorsalis. Wie man aus dieser kurzen Beschreibung des Fasciculus supraopticus ersieht, und wie ein Vergleich mit den Abbildungen 1-5 auf Tafel II der erwïhnten Abhandlung ïber Didelphis marsupialis des Genaueren ergibt, entsprechen die anatomische Anordnung des Fasciculus supraopticus und die des Tractus praeopticus einander. Ein weiterer Vergleich zwischen beiden Faserzügen ist darin gegeben, dass der Tractus praeopticus stellenweise in zwei Faserzüge zerfüllt und auch der Fasciculus supraopticus jederseits als ein geschlossener Faserzug verläuft, also auch hier eine Zweiteilung des ganzen Systems vorliegt, die bei Bufo bereits angedeutet ist. Bei anderen Amphibien zerfällt dieses Fasersystem in mehrere einzelne Züge, zeigt keine Geschlossenheit zu einem oder zu zwei Zügen. Vergleicht man den Tractus praeopticus der Amphibien mit dem Fasciculus supraopticus, so liegt weiter die Annahme nahe, dass die Ganglia supraoptica frontalia et caudalia der Marsupialia sich allmălich differenziert haben mögen aus den Zellen um den Recessus praeopticus herum, d. h. aus dem Nucleus praeopticus. Jedenfalls ergibt sich soviel, dass wir in beiden Faserzïgen alte Systeme vor uns haben. Der bei den Amphibien erhobene Befund wirft somit ein Licht auf die vergleichende Anatomie und die 
Phrlogenese der Ganglia optica basalia der Säugetiere. Sie soll des genaueren in einer besonderen Arbeit in den Folia NeuroBiologica dargestellt werden.

\section{Erklärung der Abbildungen auf Tafel II.}

Fig. 1-5. Fünf in fronto-kaudaler Richtung aufeinander folgende Frontalschnitte durch diss Gehirn von Bufo, gefärbt mit der Weigertschen IIarkscheidenfärbung.

Firg. 6. Sagittalschnitt durch den Recessus praeopticus von Bufo, gefürbt mit der Weigertschen Markscheidenfärbung.

$$
\text { Bezeichnungen. }
$$

Fig. 1. Recessus praeopticus; Nucleus praeopticus; Tractus praeopticus.

Fig. 2. Fasern des medialen Vorderhirnbündels; Nucleus praeopticus; Recessus pracopticus; Tractus praeopticus.

Fig. 3. Cellulae magnac Nuclei praeoptici: Nucleus praeopticus; Recessus praeopticus; Tractus praeopticus; Tractus opticus.

Fig. 4. Mediales Vorderhirnbündel; Laterales Vorderhirnbündel; Tractus praeopticus; Ventriculus diencephali; Chiasma.

Fig. 5. Ventriculus diencephali; Postchiasmatische Kreuzung; Ventriculus Hypothalami; Pars hypothalamica des basalen Vorderhirnbündels.

Fig. 6. Recessus praeopticus; Tractus praeopticus; Chiasma. 


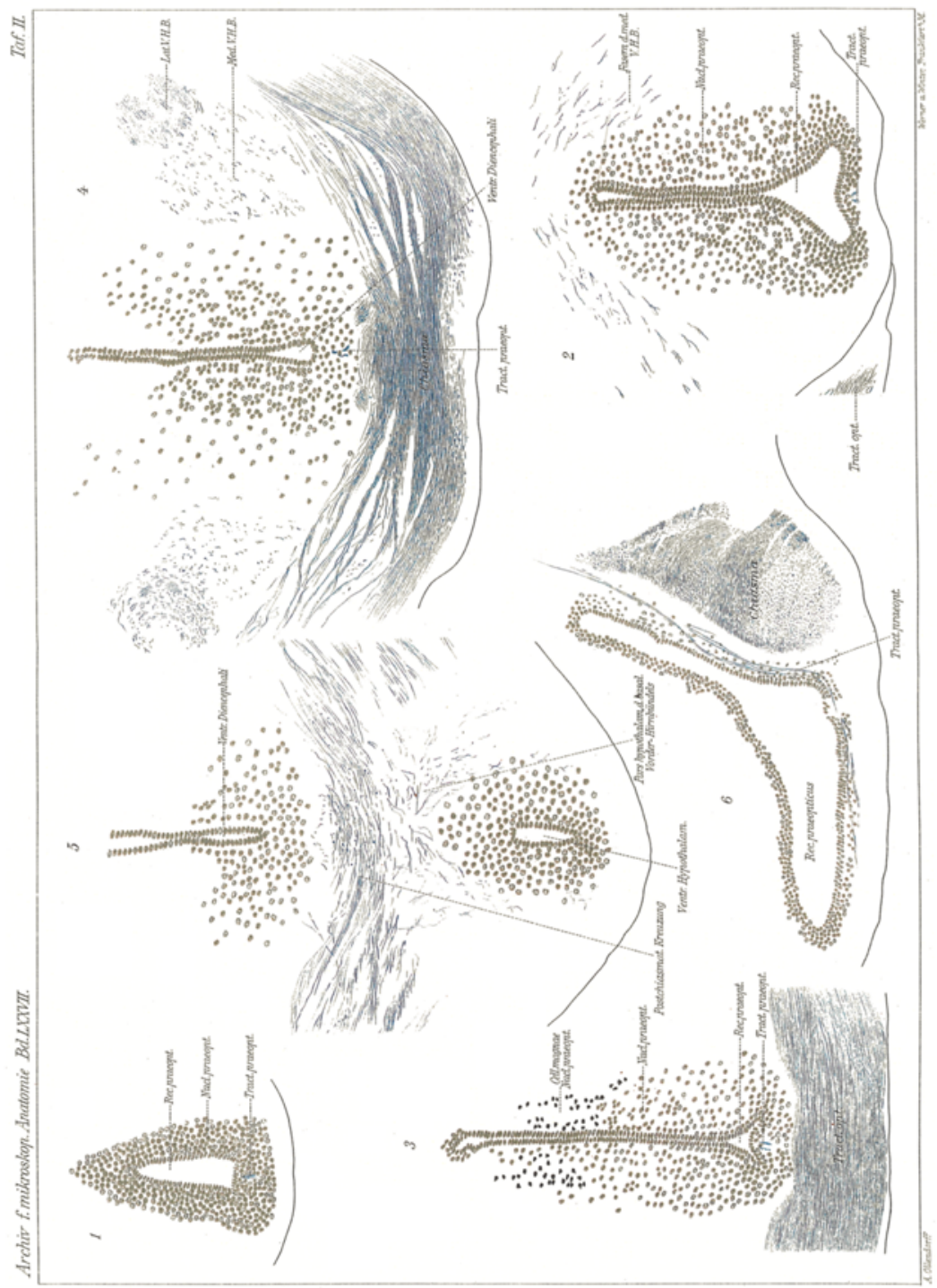

